\title{
Hierarchical star formation in M 51: star/cluster complexes
}

\author{
N. Bastian ${ }^{1}$, M. Gieles ${ }^{1}$, Yu. N. Efremov ${ }^{2}$, and H. J. G. L. M. Lamers ${ }^{1,3}$ \\ 1 Astronomical Institute, Utrecht University, Princetonplein 5, 3584 CC Utrecht, The Netherlands \\ e-mail: bastian@astro.uu.nl e-mail: bastian@astro.uu.nl, gieles@astro.uu.nl \\ 2 Sternberg Astronomical Institute of Moscow State University, Universitetsky Prospect, 13, Moscow, 119899, Russia \\ e-mail: efremov@sai.msu.ru \\ 3 SRON Laboratory for Space Research, Sorbonnelaan 2, 3584 CA Utrecht, The Netherlands \\ e-mail: lamers@phys.uu.nl
}

Received 31 March 2005 / Accepted 28 June 2005

\begin{abstract}
We report on a study of young star cluster complexes in the spiral galaxy M 51. Recent studies have confirmed that star clusters do not form in isolation, but instead tend to form in larger groupings or complexes. We use HST broad and narrow band images (from both WFPC2 and $A C S$ ), along with BIMA-CO observations to study the properties and investigate the origin of these complexes. We find that the complexes are all young $(<10 \mathrm{Myr})$, have sizes between $\sim 85$ and $\sim 240 \mathrm{pc}$, and have masses between 3-30 $\times 10^{4} M_{\odot}$. Unlike that found for isolated young star clusters, we find a strong correlation between the complex mass and radius, namely $M \propto R^{2.33 \pm 0.19}$. This is similar to that found for giant molecular clouds (GMCs). By comparing the mass-radius relation of GMCs in M 51 to that of the complexes we can estimate the star formation efficiency within the complexes, although this value is heavily dependent on the assumed $\mathrm{CO}-\mathrm{to}-\mathrm{H}_{2}$ conversion factor. The complexes studied here have the same surface density distribution as individual young star clusters and GMCs. If star formation within the complexes is proportional to the gas density at that point, then the shared mass-radius relation of GMCs and complexes is a natural consequence of their shared density profiles. We briefly discuss possibilities for the lack of a mass-radius relation for young star clusters. We note that many of the complexes show evidence of merging of star clusters in their centres, suggesting that larger star clusters can be produced through the build up of smaller clusters.
\end{abstract}

Key words. galaxies: individual: M 51 - galaxies: star clusters - galaxies: starbursts

\section{Introduction}

Recent studies of star cluster populations have shown that young star clusters do not form in isolation, but tend to be clustered themselves (Zhang et al. 2001; Larsen 2004). Despite the relatively large amount of attention that young star clusters have received in recent years, these complexes have been largely ignored, leaving many of their basic properties unknown. What triggers the formation of these complexes? What are their masses and sizes? Do their properties resemble those of giant molecular clouds (GMCs) from which they are formed or are they more akin to single young massive star clusters? It is this last question that may shed light on their formation mechanism.

Star/cluster complexes are the largest and oldest objects in the hierarchy of embedded groupings, which starts with multiple stars and finishes on galactic scales. The younger and smaller complexes are always within the older and the larger ones. This hierarchy is similar to the fractal distribution observed in the interstellar gas and in fact is the result of the latter (Elmegreen \& Efremov 1996; Efremov \& Elmegreen 1998, 2004).
Large star forming complexes associated with the spiral arms of disk galaxies are well known. Analytic considerations by Elmegreen (1994) showed that strong spiral arms will trigger GMC formation within them, which in turn may lead to the formation of star/cluster complexes. The range in age of objects within these star/cluster complexes is usually quite small ( $<20$ Myr) suggesting a coherent formation mechanism, which separates them from other large star forming regions within disk galaxies (such as the local Gould Belt in the Galaxy) which have a much larger intrinsic spread in age within them (Efremov 1995). Elmegreen \& Elmegreen (1983) suggested that complexes associated with spiral arms form from large $\mathrm{HI} / \mathrm{CO}$ clouds due to gravitational instabilities along the spiral arm. If the complexes formed directly from large gas clouds we may expect them to retain some of the properties of their progenitor clouds. Additionally, the high pressures associated with spiral arms are conducive to the formation of massive star clusters instead of loose associations (Elmegreen \& Efremov 1997). Thus we may expect complexes associated with spiral arms to contain a rich population of compact stellar clusters.

Galactic giant molecular clouds (GMCs) have a clear relation between their mass and radius, namely $M_{\mathrm{GMC}} \propto R_{\mathrm{GMC}}{ }^{2}$ 
(Solomon et al. 1987). This relation holds down to the scale of cloud clumps, which are only a few parsecs in radius (Williams et al. 1995). Young massive star clusters (YMCs), on the other hand, show a weak relation between cluster mass and radius, namely $M_{\mathrm{YMC}} \propto R_{\mathrm{YMC}}{ }^{0.1}$, with a large scatter (Zepf et al. 1999; Larsen 2004; Bastian et al. 2005a $\mathrm{a}^{1}$ ). Assuming that virialized GMCs are the progenitors of young massive clusters, this result is quite surprising because it implies that this imprint from the parent GMC (i.e. the mass-radius relation) must be erased on timescales similar to the formation timescale of the clusters. Thus, a mechanism which destroys any initial mass-radius relation must be a key ingredient in star cluster formation models (Ashman \& Zepf 2001). In this paper, we look at one scale larger than the individual star clusters, namely that of clusters of clusters, or cluster complexes.

An important question about these complexes concerns their future evolution. $N$-body simulations (Kroupa 1998) have shown that in complexes of high cluster densities, significant merging of clusters is likely. This may provide a mechanism for the formation of extremely massive clusters (Fellhauer \& Kroupa 2002; Fellhauer \& Kroupa 2005). On the other hand, Bastian et al. (2005b) have shown that gas is being expelled from complexes in the Antennae galaxies $\left(\sim 10-40 \mathrm{~km} \mathrm{~s}^{-1}\right)$. This will result in a rapid decrease in binding energy of the systems, and will tend to destroy the complexes. We will address this point by studying the properties of clusters within the complexes.

This paper is organised in the following way. In Sect. 2 we present both the optical and CO observations and introduce the complexes in M 51. Section 3 is dedicated to the derivation of the properties of each complex (e.g. their size, age, mass and star formation rates), as well as an analysis of the cluster population within each complex. In Sect. 4 we discuss the formation and evolution of the cluster complexes in light of their relation to young massive clusters and giant molecular clouds. Finally, in Sect. 5 we discuss the complexes in terms of the general hierarchy of star formation within galaxies and in Sect. 6 we summarize the main results.

\section{Observations}

\subsection{HST WFPC2 and ACS observations}

The observations used in this study were taken from the HST archive, and are presented in detail in Bastian et al. (2005a). The data set consists of two pointings, shown in Fig. 1 in Bastian et al. (2005a), each consisting of broadband $F 439 W$ $(\approx B), F 555 W(\approx V), F 675 W(\approx R), F 814 W(\approx I)$, and F656N $(\mathrm{H} \alpha)$ filters. In addition, Field 2 also has $F 336 W(\approx U)$ filter observations, which are crucial for age dating young star clusters. Thus, we will concentrate the detailed analysis on those complexes which have $F 336 \mathrm{~W}$ observations, while using the others to corroborate the results.

We have also used the Hubble Heritage ACS images which cover the entire optical galaxy in $F 435 W(B), F 555 W(V)$,

\footnotetext{
1 The data of Bastian et al. (2005a), of young star clusters in M 51, are consistent with no relation between the mass and radius.
}

$F 658 N(\mathrm{H} \alpha)$, and $F 814 W(I)$ filters. For a full review of the exposure and reduction information, see Mutchler et al. (2005). Due to the large coverage and high spatial resolution, this data will mainly be exploited to obtain size estimates of the individual sources within the complexes.

The complexes were found using flux contour cuts on the $F 439 W, F 555 W$ and $F 675 W$ images. In the regions away from the centre of the galaxy, this resulted in a fairly unambiguous selection. Complexes in the inner spiral regions were discarded from our sample, as background variations made the identification of the complexes non-trivial. The one exception to this is a large complex in the north-eastern section of the inner spiral arms, as this complex was noted in the study of Scoville et al. (2001). These authors also noted the existence of Complex G2 in their study. The complexes are identified in Fig. 1. The numbering system ( 1 or 2 ) corresponds to the two different spiral arms. Complexes with $F 336 W$ observations are B1, C2, D2, E2, F2, and G2.

The magnitude of each complex was measured with a circular aperture with a size set equal to that of the complex (see Sect. 3.1). The background was determined and subtracted using a ring with inner radius 6 pixels more than the radius of the complex, and with a width of 10 pixels. The magnitudes of the individual sources within the complexes were measured using the PSF fitting package HSTphot (Dolphin 2000). This is different from the technique used in Bastian et al. (2005a), who used aperture photometry. The change is due to the increased crowding within the complexes relative to the field.

The positions of the complexes are shown in Fig. 1, while Figs. 2 and 3 show enlarged images of the complexes without and with $F 336 W(\sim U)$-band data respectively. Additionally, we show an HST-ACS colour composite image of the largest complex in our sample (Complex G2) along with an image of the complex containing the largest super-star cluster (Complex A1) in Fig. 4, where blue, green and red represent the $F 439 W(\approx B)$, $F 555 W(\approx V)$ and $F 656 N(\approx \mathrm{H} \alpha)$ respectively.

Throughout this paper, we assume the same distance to M 51 which was used in Bastian et al. (2005a), namely $8.4 \mathrm{Mpc}$.

\subsection{Bima CO observations}

We obtained CO $(J=1-0)$ observations of M 51 from the BIMA SONG (Survey of Nearby Galaxies) survey ${ }^{2}$. The data are presented in detail in Helfer et al. (2003). As described in Henry et al. (2003), we used an image of M51 which was obtained by integrating the intensity over all velocity channels. The details of the observations and reduction of the data are given in detail in the preceding references, and thus will not be repeated here. The $\mathrm{CO}$ data were converted to physical units, namely solar masses per square parsec, using the same procedure as Henry et al. (2003) who adopted a CO-to$\mathrm{H}_{2}$ conversion factor (from antenna temperature to mass) of $2 \times 10^{20} \mathrm{H}_{2} \mathrm{~cm}^{-2}\left(\mathrm{~K} \mathrm{~km} \mathrm{~s}^{-1}\right)^{-1}$ (Strong \& Mattox 1996). Cloud sizes were determined by measuring the major and minor axes

\footnotetext{
2 The data were taken from the NASA Extragalactic Database, which can be found at http://nedwww.ipac.caltech.edu/
} 


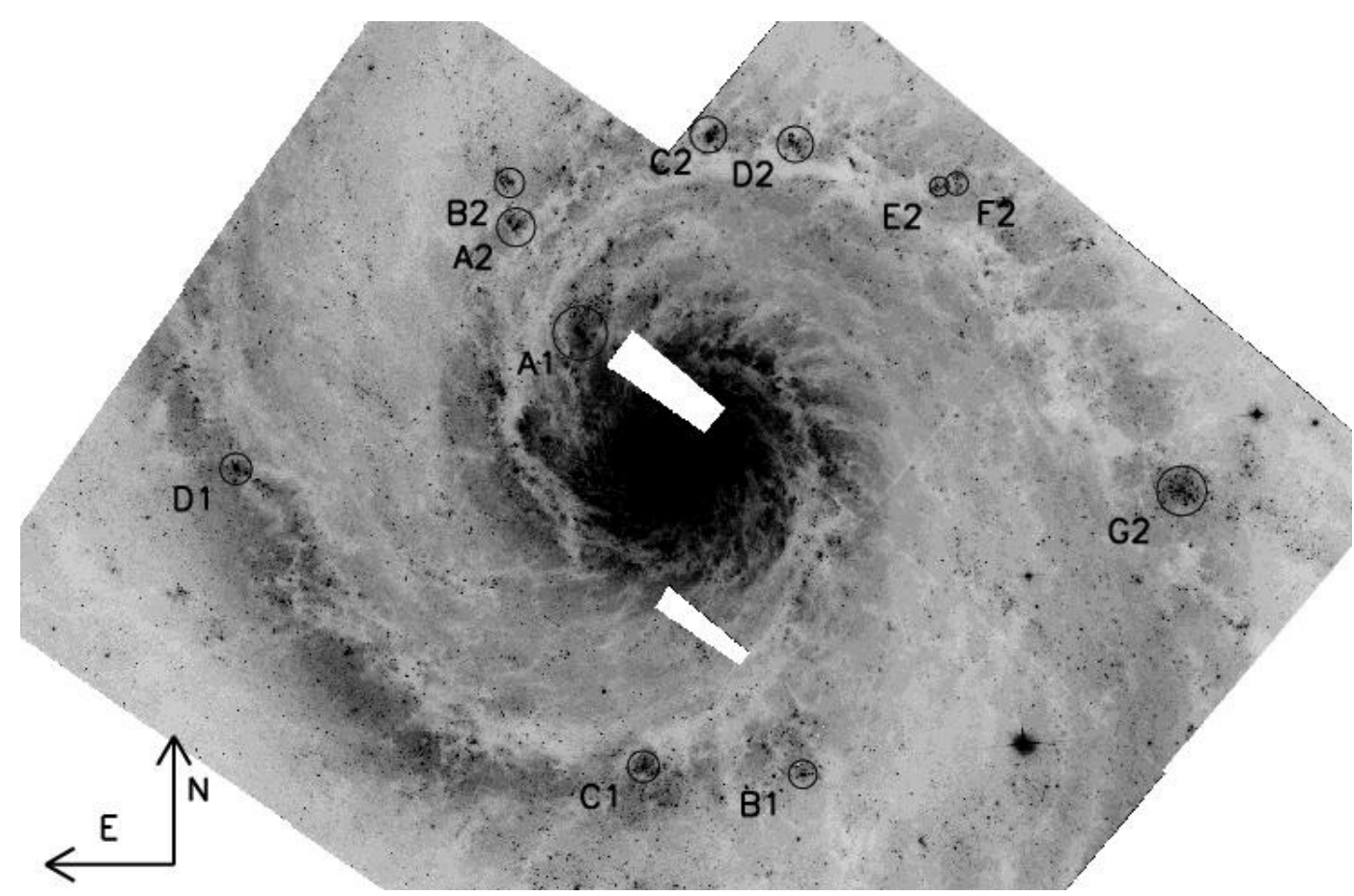

Fig. 1. Positions of the cluster complexes overlayed on the $F 555 W(V)$ band image. The complexes are identified with circles representing their measured radii as determined in Sect. 3.1. The image is $260 \times 170 \operatorname{arcsec}^{2}$.
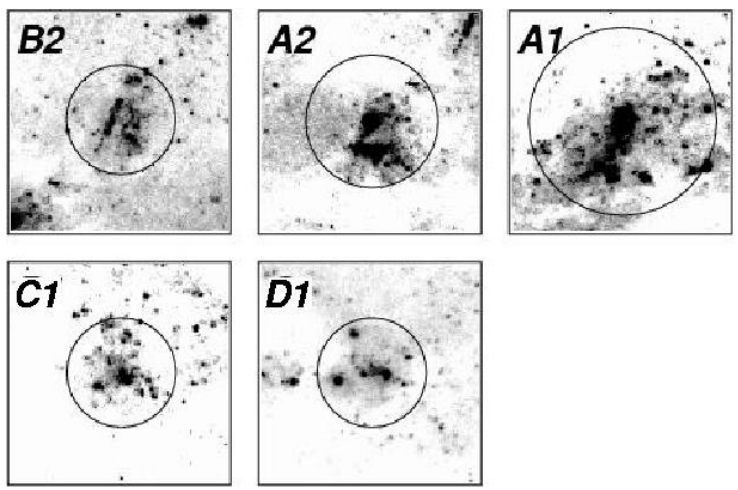

Fig. 2. $F 555 W$ images of the complexes without $F 336 W$ data. The images are $120 \times 120$ pixels $^{2}$ (12 by 12 arseconds) which corresponds to $\sim 550 \times 550 \mathrm{pc}^{2}$. The circles on the images correspond to the radius derived for each complex.

of each cloud and calculating the average. Figure 5 shows the positions of the cluster complexes on top of the BIMA intensity map.

\section{Properties of the complexes}

\subsection{Sizes of the complexes}

The size of the complexes were measured on the HST-WFPC2 images and determined through the method used for star clusters, defined by Maíz-Apellániz (2001). The method defines the edge of the complex at the point where the colour, as a function of radius, becomes constant. To determine this point, we assumed that the complexes are circular, and measured the surface brightness in concentric rings. This provides us with
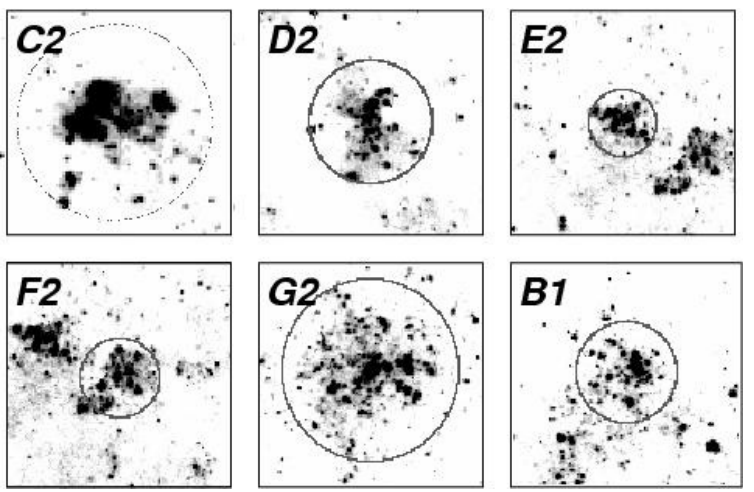

Fig. 3. $F 555 W$ images of complexes with $F 336 W$ data. The image of Complex $\mathrm{C} 2$ is $70 \times 70$ pixels $^{2}$ which is approximately 300 by $300 \mathrm{pc}^{2}$. The rest of the images are $120 \times 120$ pixels $^{2}$ ( 12 by 12 arcsec) which corresponds to $\sim 550 \times 550 \mathrm{pc}^{2}$. The circles on the images correspond to the radius derived for each complex.

surface brightness and colour profiles for each complex. An example of the flux distribution of one complex, Complex G2, is shown in Fig. 6. The vertical dashed line in the panel shows the radius adopted for this complex. The sizes of the complexes with $U$-band data $(F 336 W)$ are shown in Table 1.

It is important to note however, that the individual sources within a complex do not show any colour dependence on their position. The trend seen in Fig. 6 is based on the integrated light within each radius, which includes the background. As one looks along the radius away from the centre, the background light begins to dominate untill the measured colour is equal to that of the background, which we take as the radius of the complex. 

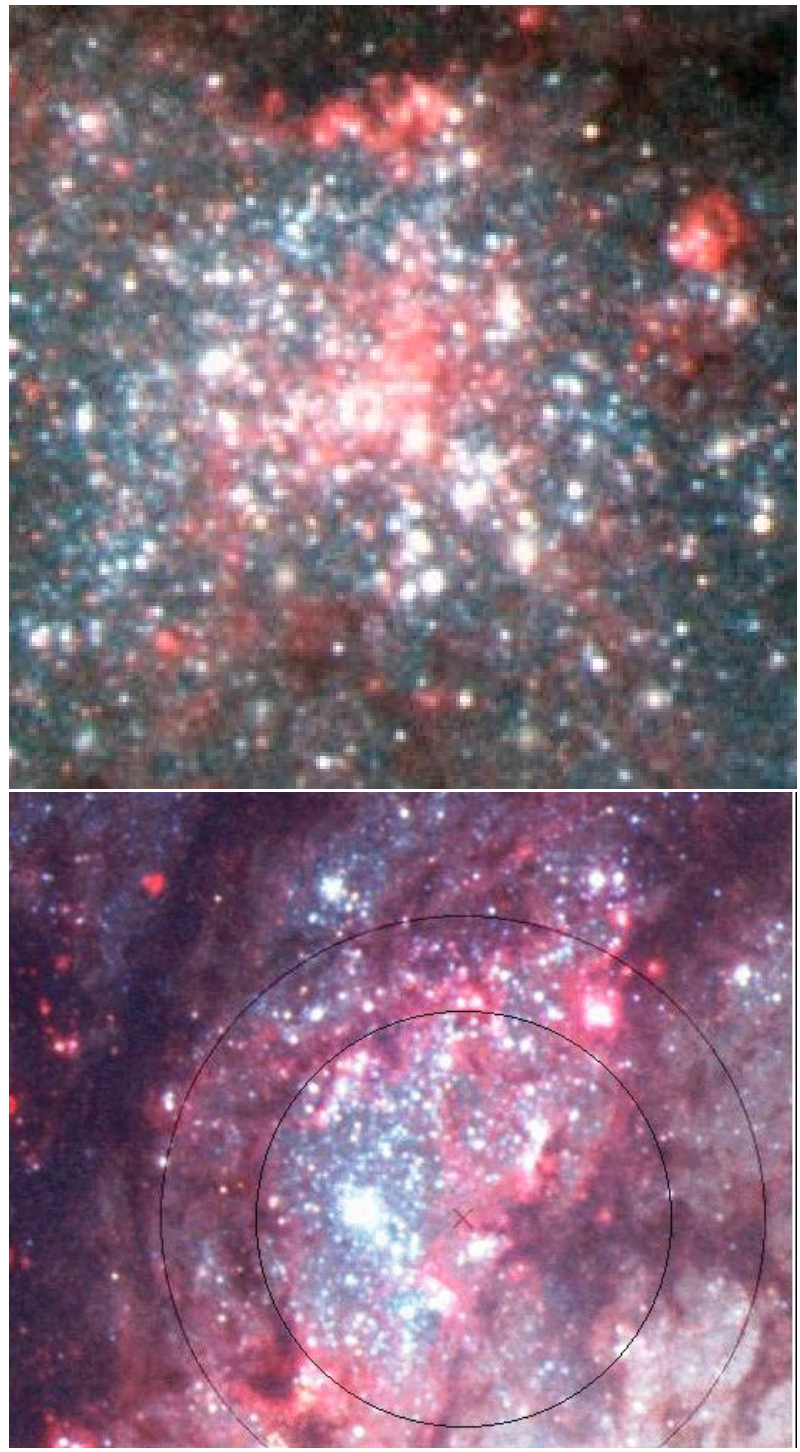

Fig. 4. Composite HST-ACS images of complex G2 (top) and A1 (bottom). The top panel is $\sim 475 \mathrm{pc}$ on a side, while the bottom panel is $\sim 800 \mathrm{pc}$ on a side. The circles in the bottom panel outline the outer and inner dust arcs, and shows that neither of them are centred on the young central star cluster (the centre of the arcs is marked by a cross). The blue, green, and red correspond to $F 439 \mathrm{~W}, \mathrm{F555W}$, and $F 656 \mathrm{~N}$ filters respectively.

\subsection{Intensity profile of the complexes}

In Fig. 6 we show the flux in concentric circles in complex G2. We have over-plotted (solid line) a function of the form, Flux $\propto r^{-0.34}$. A power-law of this form follows the data quite well (the two peaks at $\log$ radius of 1.5 and 2.0 in this distribution are due to two clusters). This corresponds to the projected density profile of a clump with $\rho(r) \propto r^{-\alpha}$, where $\alpha$ is 1.34 and $\rho$ is in units of $M_{\odot} \mathrm{pc}^{-3}$. The flux profiles of the other complexes in our sample are also well fit by power-laws of this form, with the average index, $\langle\alpha\rangle=1.74 \pm 0.34$. We will discuss the physical implications of this in Sect. 4.1.

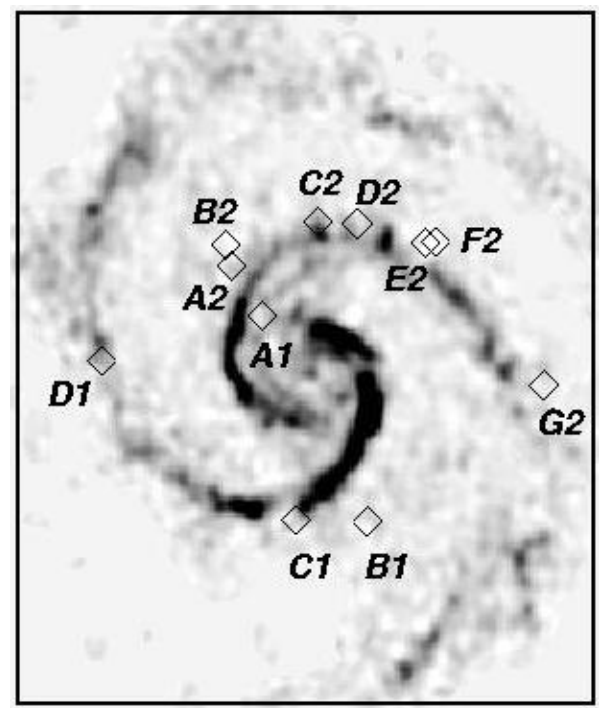

Fig. 5. BIMA CO intensity map, dark indicates regions of high intensity. North is up, and east is to the left. The positions of the cluster complexes are shown as diamonds. Note that most of the complexes fall on the outer edges of the spiral arm pattern. The lack of spatial correspondence between the complexes and high $\mathrm{CO}$ intensity regions indicates that the complexes have already destroyed their parent GMCs.

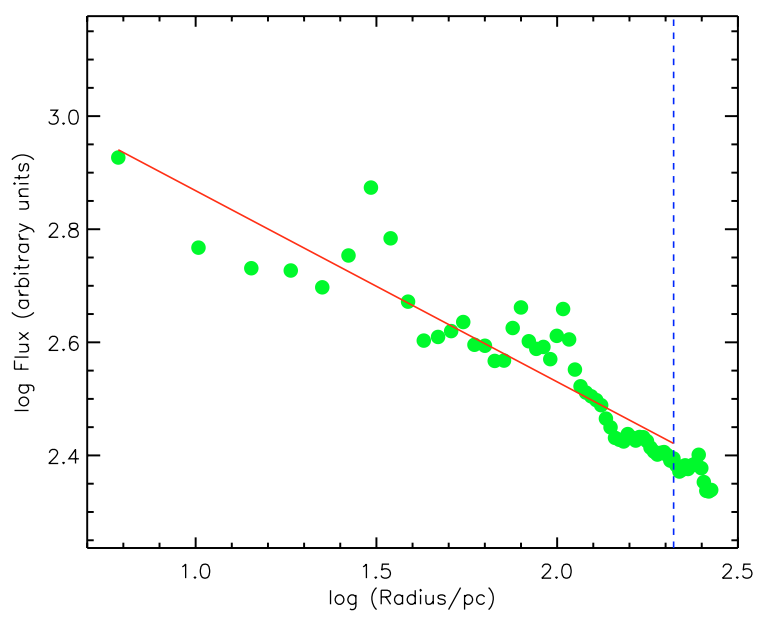

Fig. 6. The flux in concentric circles of Complex G2. The solid red line represents a profile where flux $\propto r^{0.34}$, while the vertical dashed line marks the adopted size of the complex. The two bumps in the profile are bright sources within the complex. All complexes studied here have similar power-law flux profiles (see text).

\subsection{Ages of the complexes}

\subsubsection{Ages determined by $\mathrm{H} \alpha$ measurements}

The presence of $\mathrm{H} \alpha$ emission within the complexes indicate that they are quite young, and as such suffer from the degeneracy between age and extinction (e.g. Bastian et al. 2005b). We therefore first determine the ages of the complexes (with $F 336 \mathrm{~W}$ data) using the $\mathrm{H} \alpha$ emission line width, which is independent of extinction.

To estimate the equivalent widths of $\mathrm{H} \alpha$ we use the $F 656 \mathrm{~N}$ narrow band filter. The $F 675 \mathrm{~W}$ filter was used to 
Table 1. Properties of the complexes in M 51 with $F 336 W$ and $\mathrm{H} \alpha$ imaging.

\begin{tabular}{ccccccc}
\hline \hline ID & $\begin{array}{c}M_{V}{ }^{a} \\
(\mathrm{mag})\end{array}$ & $\begin{array}{c}M_{V, \text { cluster }}{ }^{b} \\
(\mathrm{mag})\end{array}$ & $\begin{array}{c}\text { Mass }^{c} \\
\left(10^{5} M_{\odot}\right)\end{array}$ & $\begin{array}{c}\text { Radius } \\
(\mathrm{pc})\end{array}$ & $\begin{array}{c}\text { Tidal radius } \\
(\mathrm{pc})\end{array}$ & $\begin{array}{c}S F R / \mathrm{area} \\
\left(M_{\odot} \mathrm{yr}^{-1} \mathrm{kpc}^{-2}\right)\end{array}$ \\
\hline & & & & & & \\
$\mathrm{C} 2$ & -12.6 & -10.2 & 1.4 & 160 & 37 & 2.59 \\
$\mathrm{D} 2$ & -12.4 & $-9.8^{d}$ & 1.1 & 160 & 35 & 0.07 \\
E2 & -11.2 & -9.0 & 0.4 & 85 & 27 & 0.06 \\
F2 & -11.1 & -9.0 & 0.3 & 100 & 26 & 0.06 \\
$\mathrm{G} 2$ & -13.3 & -10.1 & 2.6 & 220 & 62 & 0.07 \\
B1 & -12.0 & -10.4 & 0.7 & 125 & 36 & 0.06 \\
\hline
\end{tabular}

\footnotetext{
${ }^{a}$ The magnitude of the complex within the defined radius. Uncorrected for extinction.

${ }^{b}$ The magnitude of the brightest source within each complex. Uncorrected for extinction.

${ }^{c}$ Total mass of the complex assuming an age of 7 Myr.

${ }^{d}$ Not shown in Fig. 9 because it appears to be a blend of sources, hence the colours are highly uncertain.
}

estimate the continuum contribution. Photometry was performed using apertures set to be as large as the estimated radius of each the complex. The monochromatic flux in the $F 656 \mathrm{~N}$ and $F 675 \mathrm{~W}$ band can then be found as follows: $F_{\lambda}=$ $S U M_{\lambda} *$ PHOTFLAM $_{\lambda} /$ EXPTIME $_{\lambda}$, where $\lambda$ refers to the central wavelength of the filter, $S U M_{\lambda}$ is the sum of the counts within the aperture (i.e. without background subtraction) and PHOTFLAM $_{\lambda}$ and EXPTIME $E_{\lambda}$ were taken from the image headers.

The equivalent width of $\mathrm{H} \alpha$ can now be found by

$E W(\mathrm{H} \alpha)=28.33 * \frac{F_{\mathrm{H} \alpha}-F_{R}}{F_{R}}$

where 28.33 is the rectangular width of the $\mathrm{H} \alpha$ filter in $\AA$.

The errors in $E W(\mathrm{H} \alpha)$ were estimated by calculating the $E W$ values with apertures of plus and minus 5 pixels, which is approximately the uncertainty in the radius estimates.

We can then compare the derived $E W$ for each complex, with Starburst 99 simple stellar population (SSP) models for solar metallicity and Salpeter IMF (Leitherer et al. 1999), in order to derive the age of each complex. The results are shown in Fig. 7. We note that the assumed metallicity $\left(Z_{\odot}\right.$ or $\left.2 Z_{\odot}\right)$ does not significantly affect the derived ages. We see that the complexes are indeed very young, with ages between 5 and 8 Myr. In Sect. 3.3.2 we will show that the measured cluster colours are well matched by cluster models of solar and twice solar metallicity.

This method assumes that each complex formed in an instantaneous burst, hence it puts a lower limit on the age of the complexes, as any additional formation of massive stars will tend to increase the equivalent width of $\mathrm{H} \alpha$. This means that a combination of an older burst plus new star formation can mimic the observed strength of the equivalent width.

Along similar lines, we can also look for the presence of young $\mathrm{O}$ and $\mathrm{B}$ stars at the location of the complexes. For this we compare the location of the complexes (found in the optical) to far-UV images taken by the GALEX observatory. Figure 8 shows the composite HST-Hubble Heritage image (top) as well as the composite far-UV (1530 ̊, blue) and nearUV (2310 А, red) GALEX image (bottom, taken from Bianchi et al. 2005) of the north western inner spiral arm. We find strong

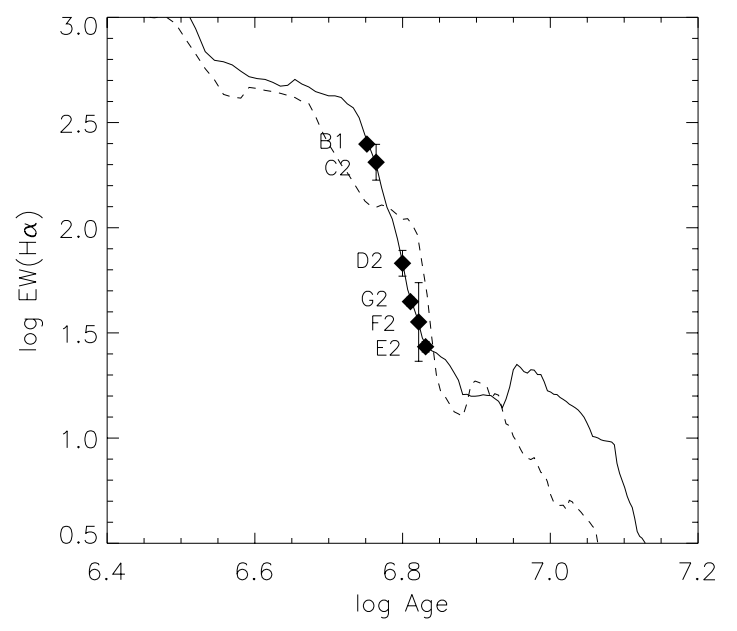

Fig. 7. Determining the ages of the complexes from the observed equivalent width of $\mathrm{H} \alpha(E W(\mathrm{H} \alpha))$ combined with the Starburst99 models (Salpeter IMF). The solid line is for solar metallicity while the dashed line is for twice solar metallicity. The $E W$ of each complex was put on the line, and the corresponding age is read off. The errors were calculated by varying the apertures used for the photometry by 5 pixels (plus and minus) around the measured radius. Note that the derived ages are largely independent of the assumed metallicity.

far-UV flux at the location of most of the complexes which is a further indication of the presence of young stars within the complexes. Also note the semi-regular spacing of star-forming regions along the spiral arm, the so-called "beads on a string" morphology. It appears that the largest isolated star-forming regions are outside the HST field of view, being located in the south-west and north-east of the galaxy. These complexes will be the subject of a future study which will exploit the full coverage of M 51 with the HST-ACS mosaic.

A comparison of Figs. 5 and 8 reveals the different stages of evolution of gas/star complexes in the NW arm, from A2 to G2. The H II complexes to the south-east of Complex C2 are seen in the UV but are not present in the $\mathrm{CO}$ data. Contrarily, the $\mathrm{H}$ II complex to the west of complex D2 is unseen in the UV but is bright in CO. Presumably, we are witnessing complexes in different stages of their evolution from $\mathrm{CO}$ complexes, to large H II regions, and finally to star/cluster complexes. 


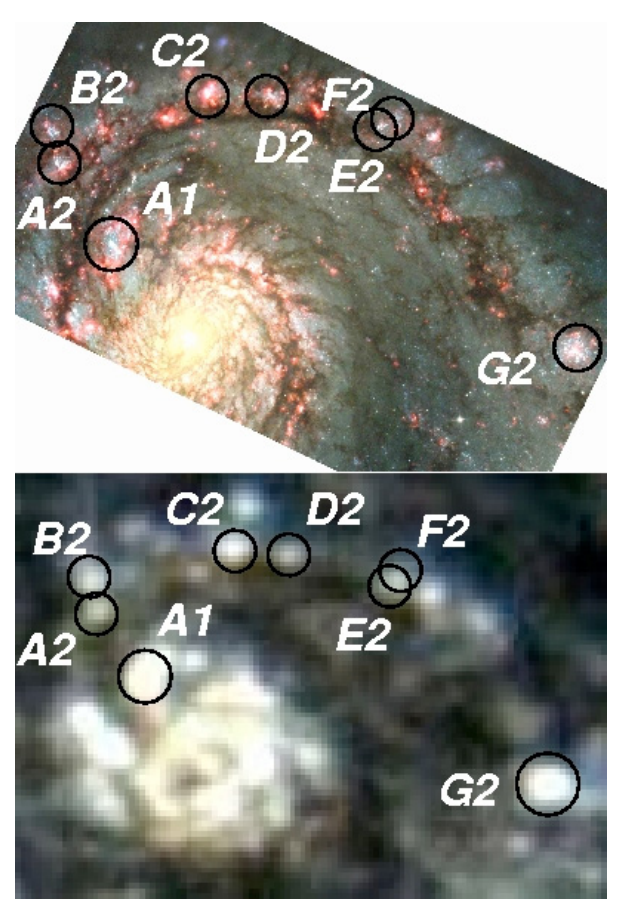

Fig. 8. Top: Hubble Heritage image of the north western inner spiral arm of M 51. Bottom: GALEX composite far-UV (blue) and near-UV (red) image of the same region. Bright regions indicate strong UV flux, indicating the presence of hot $\mathrm{O}$ and $\mathrm{B}$ stars. Note the semi-regular spacing of the complexes along the spiral arm in both images. North is up and east is to the left in this image.

Finally, we note that complex G2 is located at a break of a strong $\mathrm{CO} /$ dust lane and at the beginning of a large spur. Both of these observations may be hints into its formation. The position of G2 near the outer edge of the stellar arm might be explained by its age; however, we do not find any strong correlation between the distance from the arm edge and the age for the other complexes.

\subsubsection{Ages from the individual clusters}

We can also determine the ages of the complexes by looking at the ages of the individual star clusters within the complexes. Figure 9 shows $(F 336 W-F 439 W)$ vs. $(F 555 W-F 814 W)$ for the sources within the six complexes for which we have $F 336 \mathrm{~W}$ observations, which are essential for the age dating of young star clusters (e.g. Anders et al. 2004). We also show the solar (dashed-dotted line) and twice solar metallicity (solid line) GALEV SSP model tracks for a Salpeter IMF. The filled data points are clusters with $M_{F 555 W}<-8.6$, which are highly likely to be star clusters. The open points are the fainter sources within each complex, which may be faint clusters or individual bright stars. Assuming, for the moment, that the sources within the complex are star clusters, we see that the majority of the clusters have ages of $\sim 4-10$ Myr. The exact age is difficult to determine due to age, extinction, and metallicity degeneracies for young clusters (e.g. Bastian et al. 2005b). However, we conclude that the ages derived from the $\mathrm{H} \alpha$ equivalent widths of the complexes are consistent with the ages derived from the colours of the individual clusters.
In Fig. 9 we see that complexes C2, D2, and B1 have a relatively small amount of scatter of their points around the extinction vector, implying similar ages between all the clusters. The other complexes (E2, F2, and G2), however, contain some sources that appear much older $(>100 \mathrm{Myr})$ in the colour-colour diagram. These sources may in fact be young clusters which are heavily extincted or individual massive stars. In particular the sources in G2, show a significant amount of scatter perpendicular to the extinction vector. Taken at face value this suggests that this complex has been forming clusters for at least the last $\sim 100 \mathrm{Myr}$.

Some of the sources which appear quite old in complexes E2, F2, and G2, however, may be individual bright stars. In order to test this, in Fig. 10 we plot the absolute magnitude of each source within complex G2 (uncorrected for extinction) vs. the colour $(F 555 W-F 814 W$, roughly $V-I)$. The solid lines are $G A L E V$ SSP model tracks for a $500 M_{\odot}$ (lower) and $10^{4} M_{\odot}$ (upper) cluster. The dashed lines are stellar isochrones (with solar metallicity) of 3, 5, and $10 \mathrm{Myr}$ (from left to right respectively, Lejeune \& Schaerer 2001). Open triangles show sources which appear extended, filled stars are sources which are not resolved $(\leq 1 \mathrm{pc})$, open circles denote sources with strong $\mathrm{H} \alpha$ associated with them, and filled points are sources with no size information (either because of possible crowding or low signal-to-noise). We see that many of the objects in complex G2 are consistent with both the stellar isochrones and the cluster model tracks. We note however that there is a significant grouping of sources that would be consistent with star clusters of a few $\times 10^{2} M_{\odot}$ to $\sim 10^{4} M_{\odot}$ and ages between 4 and $10 \mathrm{Myr}$ (between -0.3 and 0.5 in $F 555 W-F 814 W$ ).

Stochastic sampling of the underlying stellar IMF can cause significant deviations from standard SSP model colours (e.g. Dolphin \& Kennicutt 2002), therefore it is not completely unexpected that sources do not lie directly on the SSP model tracks. We also note that due to the large crowding in these complexes, accurate photometry is difficult and will tend to increase the scatter in diagrams such as Fig. 10. Without higher resolution imaging or spectroscopy of individual sources, the degeneracy between stars and star clusters is extremely difficult to break.

Based on the brightness criterion of $M_{V}<-8.6$, we conclude that there are clusters within the complexes and that their ages are between 4 and $\sim 10 \mathrm{Myr}$, although complexes G2, E2, and F2 seem to also contain older clusters.

It is interesting to note the existence of spatially resolved massive star clusters within the complexes, the most massive of which is located at the centre of complex A1. Using the available $H S T$-ACS images, we find that this cluster has a magnitude of $m(F 555 W)_{\mathrm{ACS}}=18.3$, and a colour $m(F 435 W)-$ $m(F 555 W)=0.32$. This, together with the strong $\mathrm{H} \alpha$ emission, is consistent with the cluster being quite young and extincted. Using the same model and distance assumptions used throughout this work, and an assumed age of the cluster of $7 \mathrm{Myr}$, we find that this cluster has a mass of $\sim 10^{5} M_{\odot}$, which is a lower limit as we have not corrected for extinction. Using the ISHAPE routine of Larsen (1999), we find this cluster to be well resolved, although significantly flattened. Using a Moffat profile with index 1.5 , we find the $F W H M$ along the major axis 

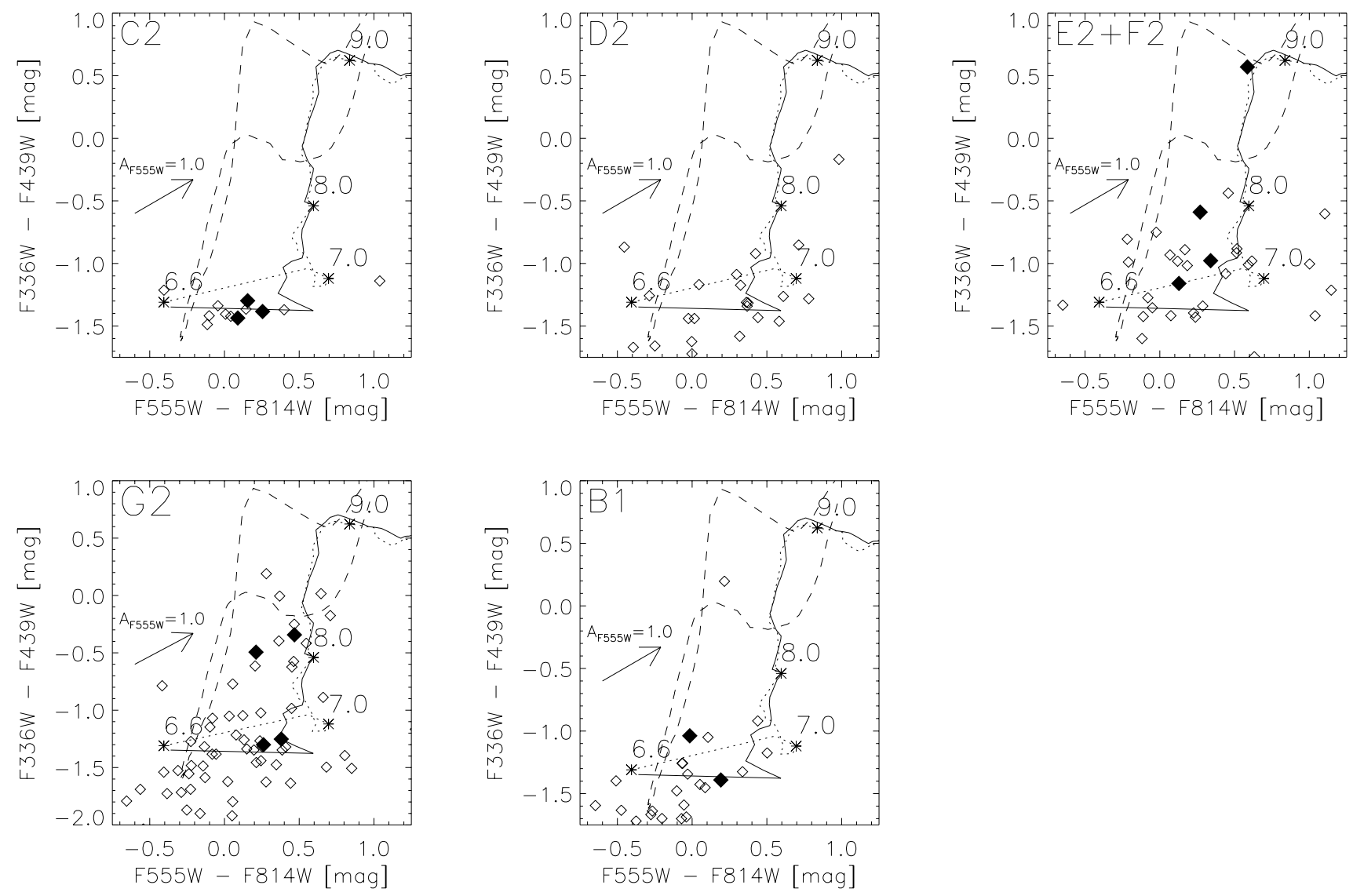

Fig. 9. Colour-colour diagrams for sources in the six cluster complexes with $F 336 \mathrm{~W}$ observations. The names of the complexes are given in each panel. Filled points are for sources with $M_{V}<-8.6$ (uncorrected for extinction), while open points are fainter than this (somewhat arbitrary) limit. The solid line represents the GALEV SSP models for twice solar metallicity while the dotted line represents the same models but for solar metallicity. The asterisks mark age points on the evolutionary curves, labelled as the logarithm of the age in years. The long dashed line is a stellar isochrone for a solar metallicity, $10 \mathrm{Myr}$ old, population (however the isochrone for twice solar metallicity is extremely similar in this region of colour space). The arrow in each panel represents the extinction correction of $A_{F 555 W}=1.0$.

of the cluster to be $2.6 \pm 0.5 \mathrm{pc}$, and a major to minor axis ratio of 0.6. In a future work, we will present an in depth analysis of the cluster and stellar populations within each complex. Another interesting feature of this complex, are two dust arcs which appear to have the same centre, which is displaced from the young central massive star cluster (shown in the bottom panel of Fig. 4). The origin of these arcs remains unclear.

\subsection{Masses of cluster complexes}

The masses of the complexes were estimated by combining the complex luminosities and the age dependent mass-to-light $(M / L)$ ratios of the GALEV SSP models. We assign all complexes the same age, $\sim 7 \mathrm{Myr}$, and interpolate the SSP models between 4 and $8 \mathrm{Myr}$ to determine $M / L$ for this age. We have used the $F 555 \mathrm{~W}$ magnitudes to derive the masses of the complexes, but we note that the other broad-band magnitudes give similar results. The masses of these complexes range from $0.3 \times 10^{5} M_{\odot} \quad(\mathrm{F} 2)$ to $3.0 \times 10^{5} M_{\odot} \quad(\mathrm{G} 2)$ and are given in Table 1. The errors on the determined masses (as seen in Fig. 12) are based on errors in the age estimate, from 4 to $12 \mathrm{Myr}$.

\subsection{Star formation rates within the complexes}

Using the continuum subtracted $\mathrm{H} \alpha$ images, we can estimate the star formation rates, $\Sigma_{\mathrm{SFR}}$, within the complexes. For this, we adopt the conversion factor of $\mathrm{H} \alpha$ luminosity to star formation rate of Kennicutt (1998a), namely

$$
\Sigma_{\mathrm{SFR}}\left(M_{\odot} \mathrm{yr}^{-1}\right)=7.9 \times 10^{-42} L(\mathrm{H} \alpha)\left(\mathrm{ergs} \mathrm{s}^{-1}\right) .
$$

The values derived are shown in Table 1. The star formation rates per unit area of the complexes are comparable to the definition of a starburst galaxy $\left(0.1 M_{\odot} \mathrm{yr}^{-1} \mathrm{kpc}^{-2}\right.$, Kennicutt et al. 2004), justifying complex C2 as a localized starburst (terminology from Efremov 2004). Other complexes from Table 1 have lower SFRs.

Star cluster complexes in the Antennae galaxies have area normalised star formation rates which are approximately 10 times higher than the ones studied here, despite having similar sizes (Bastian et al. 2005b). This difference can be understood by comparing the GMCs properties in the two galaxies. The resolved GMCs in the Antennae (Wilson et al. 2003) have roughly 10 times the mass, and hence 10 times the density, as those in M 51 for a given spatial size. Therefore, the differences in the SFR/area can be well explained through the Schmidt law 


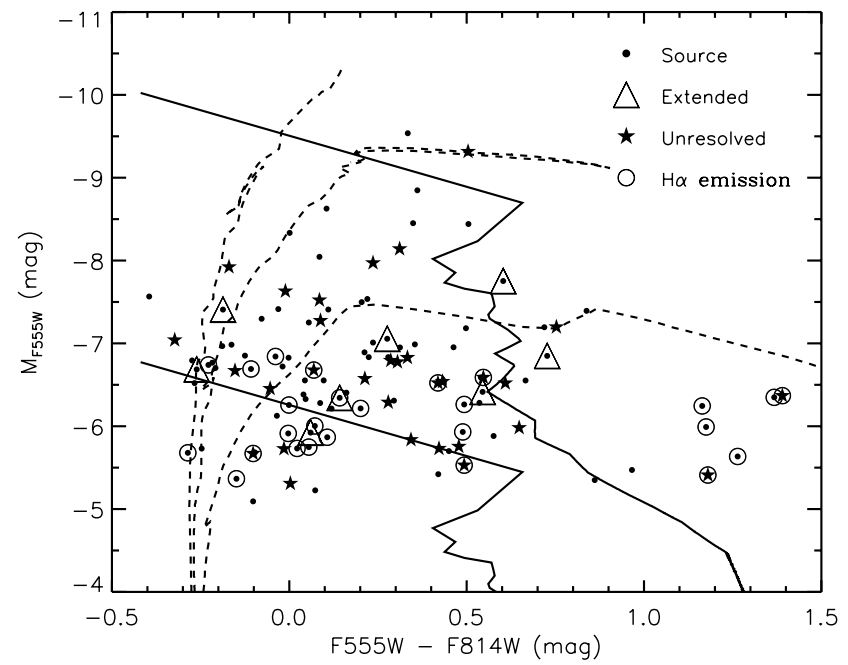

Fig. 10. Colour-magnitude diagram of the sources within complex G2 (from the ACS images). The dotted lines are solar metallicity stellar isochrones for 3, 5, and $10 \mathrm{Myr}$ from left to right (the twice solar metallicity isochrones occupy the same region in this figure). The solid lines are GALEV SSP (cluster) model tracks (Salpeter IMF and twice solar metallicity) for a $10^{4} M_{\odot}$ (upper) and $500 M_{\odot}$ (lower) cluster. The open triangles denote sources which appear extended, the stars are sources which are not resolved $(\leq 1 \mathrm{pc})$, open circles are sources which have strong $\mathrm{H} \alpha$ emission associated with them, and filled dots are detected sources which have no size information (due to possible crowding or low $\mathrm{S} / \mathrm{N}$ ).

(Schmidt 1959) where the rate of star formation is proportional to the gas density to the power $N$. Kennicutt (1998b) has shown that $N \sim 1.4 \pm 0.15$ for normal disk galaxies.

\section{Comparison between the properties of cluster complexes and GMCs}

\subsection{The density profiles}

In Sect. 3.2 we found that the surface brightness of complex G2 is well fit by a power-law of the form Flux $\propto r^{-0.34}$. Assuming sphericity, this corresponds to a three dimensional density profile of $\rho \propto r^{-\alpha}$, with $\alpha=1.34$. The other complexes were also shown to be well fit with profiles of this type, with $\langle\alpha\rangle=1.74 \pm 0.34$. This power-law profile is similar to that observed for GMCs ( $\alpha=1-2$, Ashman \& Zepf 2001) $)^{3}$ and much shallower than King profiles which fit old globular clusters quite well. The similarity between the profiles of GMCs and the cluster complexes studied here suggests that the amount of luminous material formed is proportional to the gas density at that point within the GMC. We shall return to this point in Sect. 5.1. Throughout the present work we assume that the complexes are spherical, which in the $z$-axis would make the complexes larger than the thickness of typical galactic disks. This is also true for the GMCs. If both the GMCs and the complexes are truncated in the vertical direction (i.e. flattened) our main conclusions would still remain valid.

\footnotetext{
${ }^{3}$ Recent studies have shown that Galactic GMCs have $\alpha=1.7 \pm$ 0.2, Joao Alves priv. comm.
}

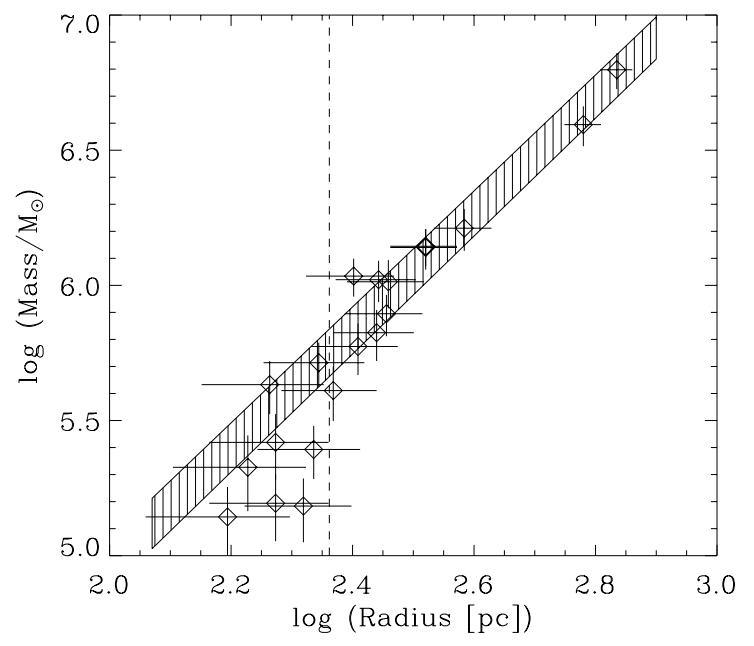

Fig. 11. The mass vs. radius relation for GMCs in M 51. The vertical dashed line indicates the spatial resolution of the BIMA survey. The filled area is a power-law fit to the data above the resolution limit, with index 2.16, and the corresponding $1 \sigma$ error bars on the index $( \pm 0.20)$ and the zero point.

\subsection{Size and radius relation of GMCs and cluster complexes}

By combining the study of the cluster complexes and GMCs in M 51 we can gain insight into the hierarchical nature of structure formation within spiral galaxies. Solomon et al. (1987) showed that there is a clear relationship between size and mass for GMCs in the Galaxy, namely $M_{\text {Cloud }} \propto R_{\text {Cloud }}^{2}$, a consequence of virial equilibrium. This relation has also been found for GMCs outside the Galaxy (see summary in Ashman \& Zepf 2001).

However, as shown here, a single massive GMC does not produce a single star cluster, but a complex of star clusters. Therefore it is interesting to see how these complexes fit into the hierarchy of star formation. We begin by searching for a relation between mass and radius for GMCs in M 51 . Figure 11 shows the radius vs. mass for the detected GMCs within M 51. The dashed vertical line shows the spatial resolution of the BIMA survey. We have fit a function of the form $\log M=\kappa \cdot \log R+c$ (i.e. $\left.M \propto R^{\kappa}\right)$. We find that $\kappa=2.16 \pm 0.20$. The filled area corresponds to the best fit to the data above the resolution limit including the $1 \sigma$ error (this area also includes the error on the zero point of the function).

Figure 12 shows the result of the same analysis applied to the cluster complexes. Here we find that $\kappa=2.33 \pm 0.19$. The filled area with the horizontal hash marks represents the best fit plus the corresponding $1 \sigma$ errors. The filled area with the vertical hash marks is the fit (plus errors) of the GMCs extrapolated into the size range of the complexes. We see that the relations for the GMCs and the cluster complexes have almost the same slope, but are offset by $\sim 0.3$ dex in the vertical (mass) direction. This offset is related to the star formation efficiency which will be discussed in Sect. 4.3.

We conclude that the cluster complexes follow a similar mass-radius relation as GMCs. On the contrary, young star clusters do not follow this mass-radius relation (e.g. 


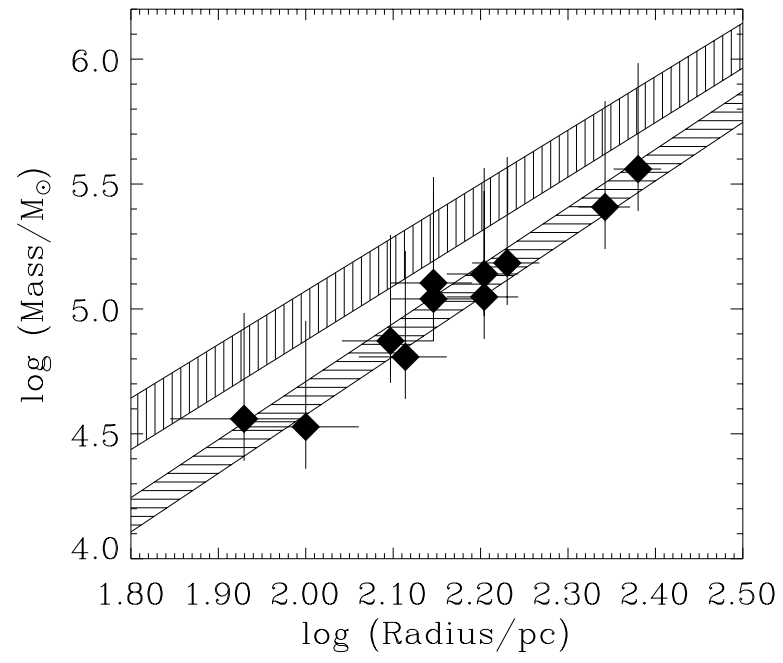

Fig. 12. The mass vs. radius relation for cluster complexes in M 51 . The solid line is a power-law fit to the data with index, 2.3. The filled area with vertical hashes is the fit to the GMCs from Fig. 11, extended into the size regime of the complexes. The filled area with the horizontal hash marks is the power-law fit to the complexes, with index 2.33, and the corresponding $1 \sigma$ error bars on the index $( \pm 0.19)$ and the zero point.

Bastian et al. 2005a). This implies that the mass-radius relation must be broken below the scale of the complexes. If binding energy is the factor that determines the star formation efficiency (assuming that the star formation efficiency causes the lack of a mass-radius relation in young star clusters) then it is not the binding energy of the cloud which is important but the binding energy of the clumps within the cloud which is the dominant factor.

Additionally we note that the mass-radius relation holds for clumps within GMCs, down to the resolution limit $(\sim 0.5 \mathrm{pc}$ and $\sim 13 M_{\odot}$ in the Rosette molecular cloud (Williams et al. 1995). This suggests that the mass-radius relation is broken during (or after) the star formation process.

\subsection{Star formation efficiency within the complexes}

We have shown that the GMCs and the star cluster complexes in M 51 share a very similar mass vs. radius relation. This offers a unique opportunity to investigate the star formation efficiency, $\epsilon=M_{\text {complex }} / M_{\mathrm{GMC}}$, for each complex. Although we cannot measure the mass of the GMC that formed the present complexes, if we assume that the parent GMCs had the same size as the complexes, we can estimate the progenitor mass. Thus it remains to be shown that the radius of the clouds do not change significantly during the formation of the complexes.

We can estimate the timescale on which the GMCs will shrink by calculating their free fall time. The free fall timescale is $t_{\mathrm{FF}} \approx(\rho \cdot \mathrm{G})^{-\frac{1}{2}}$. Using the relation between the mass and radius of the clouds derived above, we see that the free fall timescale of GMCs, as a whole, is between 80 and $200 \mathrm{Myr}$. The ages (e.g. the upper limit of the formation timescale) of the complexes are between 5 and $10 \mathrm{Myr}$, much shorter than the collapse time of the GMCs. This means that the complexes formed from the GMCs on a timescale much shorter than the collapse time of the GMC. Thus the size of the complexes should be about the same as that of the GMC from which they formed.

In Fig. 12 we show the mass-radius relation for the complexes (the data points along with the best fitting power-law relation with the associated $1 \sigma$ errors) and over-plot the relation for the GMCs, extrapolated to the size scale of the complexes. The vertical offset between the best fit line of the complexes and the best fit line of the the GMCs is the star formation efficiency, $\epsilon$. In the case of M 51, we measure $\epsilon$ of the complexes to be $50 \pm 20 \%$. The convergence of the two relations at higher masses suggests that there is a trend for $\epsilon$ to increase for larger complexes, but the trend is well within the observational errors.

An additional caveat to this approach is that we have assumed the mean Galactic CO-to- $\mathrm{H}_{2}$ conversion factor. However, studies of M 51 have shown that the conversion factor in M 51 is roughly half that of the Galaxy (Boselli et al. 2002). Using this value would make GMCs half as massive as that estimated here, and therefore the star formation efficiency would be close to $100 \%$. A final caveat to this estimate is the assumed stellar initial mass function for the complexes. We have assumed a Salpeter IMF from 0.1 to $50 M_{\odot}$, however if we would assume a Kroupa-type stellar IMF, the masses of the complexes would be lowered by a factor of $\sim 2$, giving $\epsilon=\sim 25 \%$. Therefore we are left to conclude that while this method to estimate the star formation efficiency is intriguing, it is limited at the present time by the assumptions that are required.

However, it should be noted that the assumed CO-to- $\mathrm{H}_{2}$ factor will not effect the index of the mass-radius relation of the GMCs presented here. The GMCs in M 51 used in this study are all located within the inner $5 \mathrm{kpc}$ of the galaxy, and therefore we do not expect much cloud to cloud variation in the conversion factor, as the metallicity is not expected to change significantly over this area.

\section{Discussion}

\subsection{Formation of the complexes}

We can compare the properties of the complexes in M 51 with those of complexes and young/old star clusters in the Antennae galaxies which have been measured by Whitmore et al. (1999). Knot $\mathrm{S}^{4}$ in the Antennae galaxies has an extremely similar power-law profile (Flux $\propto r^{-1 / 2}$ ) over $\sim 300$ pc from the centre as complex G2. Additionally, the very young $(<10 \mathrm{Myr})$ massive star cluster \#430 in the Antennae follows the same power-law density distribution. However, the older cluster \#225 ( $\sim 500$ Myr old) does not follow a clear power-law relation but instead has a sharp cut-off at $\sim 50$ pc. Presumably cluster \#225 was formed with a power-law density profile which has been eroded due to dynamical evolution. Additional support for this scenario comes from star clusters in the LMC, where young

\footnotetext{
4 The large amount of substructure within this knot, particularly in the $F 555 W(\approx V)$ and $\mathrm{H} \alpha$ bands, suggests that this knot is a cluster complex, and not just a single large star cluster.
} 
star clusters show a power-law density profile (with no distinct cut-off radius) (Elson et al. 1987) while older clusters have King-type profiles with a distinct tidal truncation.

The similarity between the profiles of GMCs and the complexes studied here suggests that the amount of luminous material formed at a certain radius within a GMCs is proportional to the density of the cloud at that radius. This naturally explains why the complexes share the same mass-radius relation as GMCs (see Sect. 4.2), as any relation inherent to the progenitor cloud will be imprinted onto the complexes as they form. Additionally, the similarity between the projected profiles of the complexes, and young clusters implies a common formation mechanism (i.e. star formation proportional to the gas density).

However, young cluster systems, such as those of NGC 3256 (Zepf et al. 1999), M 51 (Bastian et al. 2005a), and various spiral galaxies (Larsen 2004) have a weak relation between their mass and radius (with a large scatter). The similarity between the density profiles of the complexes and young star clusters makes the lack of a mass-radius relation in star clusters all the more surprising. This is because we would also expect them to bear the imprint from the cloud of which they formed.

Ashman \& Zepf (2001) have suggested that a star formation efficiency which depends on the binding energy (predicted by Elmegreen \& Efremov 1997) of the progenitor GMC could destroy such a relation during the formation of young clusters. But this theory does not explain the shallower size distribution of star clusters relative to GMCs.

Another possible explanation for this lack of a mass-radius relation in young clusters is that dynamical encounters between young clusters (and gas clouds) add energy into the forming clusters, thereby increasing their radii. Some support for this scenario is given by differences in the size distributions of clusters and GMCs. GMCs (e.g. Elmegreen \& Falgarone 1996) and cloud clumps (e.g. Williams et al. 1995) follow the size distributions of $N(r) \mathrm{d} r \propto r^{-\eta} \mathrm{d} r$ where $\eta \approx 3.2$. Star clusters (both young and old), however, follow a shallower relation, namely a power-law with $\eta \approx 2.2$ (Bastian et al. 2005a). If the protoclusters follow the same mass-radius relation as GMCs and clumps, then their density will be $\rho \propto M / R^{3} \propto R^{-1}$, showing that the larger proto-clusters are less dense. Due to their lower density, we expect larger proto-clusters to be more affected by encounters, i.e. making large clusters even larger. This would tend to make the size distribution shallower, as observed.

\subsection{Truncation of the size of the complexes}

The complexes most closely associated with spiral arms (e.g. excluding complexes $\mathrm{C} 1$ and $\mathrm{G} 2$ ) have diameters similar to the minor axes of GMCs within the arms. We have shown that the size of a complex does not change significantly from the progenitor GMC. This suggests that the maximum size (and hence mass due to the mass-radius relation) of the complexes is determined by the size of the GMCs within the galaxy. Due to sheer effects, caused by the flat rotation curve of the disk, GMCs have a maximum size (hence mass). This in turn will determine the maximum sized complex which can form. If the star clusters within the complex are formed with an initial mass function, then the most massive cluster formed will be dependent on the size of the parent complex. There does seem to be a relation between the most massive GMC and the most massive star cluster within a galaxy (e.g. Wilson et al. 2003).

However, there are two exceptions where the size of a complex is larger than the typical semi-minor axis of a GMC. These two complexes (A1 and G2) do not seem to be directly related with a spiral arm, in fact A1 is on the inside of the spiral arm. G2 is located at the starting point of a large spur, which is a sign of instabilities in the arm. Instabilities, such as large spurs, may allow larger GMCs to exist for short periods, and hence explain the existence of these larger than expected complexes.

\subsection{The evolution of the cluster complexes}

As shown in Sect. 3.2, the complexes studied here are all quite young. However, if the complexes are a long lived phenomenon, then we would expect to see complexes throughout the disk and not just associated with the spiral arms. Elmegreen (1994) predicted that the remnants left over from "superclouds" within the spiral arms, (i.e. what we call complexes) should be observable as loosely bound stellar complexes in the interarm region of the galaxy. Do such inter-arm complexes exist? The present data set is not adequate to conclusively answer this question. As shown in Bastian et al. (2005a) the detection limit imposed by the data severely limits which clusters (e.g. how bright and hence how massive) we can observe. At an age of $\sim 100 \mathrm{Myr}$, we can only detect clusters (with no extinction) which have masses above $10^{4} M_{\odot}$. Therefore, we would miss smaller clusters belonging to the same complex, if they exist. We have found 3 candidate complexes with ages between 40 and $80 \mathrm{Myr}$ as determined by the colours of the detected clusters. Deeper images are necessary to resolve this question.

In order to estimate whether or not the complexes studied here are gravitationally stable, we can compare their present radii with their estimated tidal radii. In a rotating disk, this can be estimated by

$r_{t}=\left(\frac{G M_{\text {complex }}}{2 \cdot V_{G}^{2}}\right)^{1 / 3} R_{\mathrm{G}}^{2 / 3}$

where $M_{\text {complex }}, V_{G}$, and $R_{G}$ are the mass of the complex, the circular velocity of the galaxy at that point, and the distance to the galactic centre. If we assume a disk rotation velocity of $200 \mathrm{~km} \mathrm{~s}^{-1}$ (e.g. Rand 1993 for galactocentric distances greater than $1 \mathrm{kpc}$ ) and distances to the galactic centre between 2 and $4 \mathrm{kpc}$, then we see that complexes with masses between $10^{4} M_{\odot}$ and $10^{5.5} M_{\odot}$ have tidal radii of 50 to $100 \mathrm{pc}$. In Table 1 we compare the measured sizes of the complexes with their derived tidal radii. We see that the tidal radii are much smaller than the measured radii, indicating that the outer material of the complexes are not bound to the complex. Because of the steep density profile of the complexes (see Fig. 6) the inner region may be bound and survive for extended periods. If this is the case, then the inner clusters are expected to merge within a few $\times 10^{7}$ years (e.g. Kroupa 1998; Fellhauer \& Kroupa 2002), forming a single massive star cluster like object. 
Elmegreen et al. (2000) and Larsen et al. (2002) have speculated that the most massive cluster within the giant stellar complex in NGC 6946 may have formed by the merging of other members of the complex. This cluster is located in the centre of the complex and its measured age (through photometry and spectroscopy) appears to be the average of the surrounding clusters, which is exactly as the merger theory predicts. We note that the centres of many of the complexes presented here, show the presence of massive and elongated objects, in particular complexes A1, E2, F2, and G2. We take the presence of such objects as evidence for merging in the centre of the complexes. Higher resolution imaging should offer more definitive evidence of on-going merging in the centres of these complexes.

\section{Summary and conclusions}

1. Cluster complexes in M 51: from archival HST imaging we identified 9 cluster complexes in the inner $\sim 5 \mathrm{kpc}$ of M 51. We derived their ages by comparing their equivalent widths of $\mathrm{H} \alpha$ to that of Starburst99 SSP models. We checked the validity of these age measurements by comparing the colours of the star clusters within each complex to the GALEV SSP models. We find reasonable agreement between the two methods.

2. GMCs in M51: we have also measured the sizes and masses of a sample of giant molecular clouds in M 51 from existing CO data. This was done in order to enable a comparison between the complexes and the gas content of the galaxy.

3. The star formation rate within the complexes: we have measured the star formation rates within the complexes from the (continuum subtracted) $\mathrm{H} \alpha$ flux using the relation between the $\mathrm{H} \alpha$ flux and the star formation rate given by Kennicutt (1998a). The area normalised star formation rates are as high as starburst galaxies, thereby justifying their designation as localized starbursts, a label first coined by Efremov (2004). We found that the star formation rates (for a given size) within the complexes studied here are about a factor of 10 lower than complexes (of the same size) in the merging Antennae galaxies. We trace this difference to the molecular cloud populations within the two galaxies, as the GMCs in the Antennae galaxies are roughly 10 times more dense than the GMCs in M 51. Thus, if star formation is proportional to gas density, as is the case in the commonly used Schmidt law of star formation, then the difference in the star formation rates between the two galaxies is readily understandable.

4. The surface density distribution: the cluster complexes follow the same surface density distribution as that of GMCs and young star clusters, namely a power law with exponent $-0.74 \pm 0.34$, corresponding to a spatial density $\rho \propto r^{-1.74}$. This provides a natural explanation for the similarity between the observed mass-radius distribution of GMCs and cluster complexes. Presumably older clusters follow a steeper density distribution with a distinct tidal truncation due to dynamical evolution.
5. The mass-radius relation: the complexes in M51 follow the same mass-radius relation observed for GMCs. We conclude that this similarity is due to the imprint of the progenitor GMC onto the complex forming within it. The shared mass-radius relation is a natural consequence of the shared density distributions. Contrary to the complexes however, young star clusters do not share the same mass-radius relation (they follow a much weaker relation with a large scatter). We suggest that this may be due to interactions between the young star clusters and gas clouds. This scenario is supported by the observation that the size distribution is significantly shallower for star clusters (young and old) than for GMCs (Bastian et al. 2005a).

6. Star formation efficiency: we have argued that the size of the progenitor GMC and that of the each complex are the same, due to the short formation timescale relative to the free-fall timescale of the GMC. We estimate the star formation efficiency within each complex, by extending the observed mass-radius relation of GMCs to that of the scale of the complexes. In this way, we estimate that the star formation efficiency in the complexes is $50 \%$. However, due to the required assumptions (CO-to- $\mathrm{H}_{2}$ conversion factor and stellar IMF of the complex) the errors using this method are quite substantial, and hence this method is not viable at the present time.

7. Evolution of the complexes: the complexes studied here are quite similar to those modelled by Fellhauer \& Kroupa (2002, 2005). Their models show that a significant amount of merging of the individual clusters is expected to happen within the complexes, which may lead to the formation of a much larger star cluster than would be expected from statistical sampling of a cluster initial mass function. This may explain why clusters with masses up to $5 \times 10^{5} M_{\odot}$ are found in M51 (Bastian et al. 2005a), which is about the mass of the largest complex analysed here. Many of the complexes studied here show evidence of merging in their centres.

Acknowledgements. This research has benefited from the NASA Extragalactic Database. We thank Sren Larsen and Pavel Kroupa for useful and stimulating conversations. We would like to thank Alice Quillen for help in analysing the BIMA data. Yu.N.E. acknowledges support from grants RFFI 03-02-16288 and NSh 389-2003-2.

\section{References}

Anders, P., Bissantz, N., Fritze-v. Alvensleben, U., \& de Grijs, R. 2004, MNRAS, 347, 196

Ashman, K. M., \& Zepf, S. E. 2001, AJ, 122, 1888

Bastian, N., Gieles, M., Lamers, H. J. G. L. M., Scheepmaker, R. A., \& de Grijs, R. 2005a, A\&A, 431, 905

Bastian, N., Emsellem, E., Kissler-Patig, M., \& Maraston, C. 2005b, $\mathrm{A} \& \mathrm{~A}$, in press

Bianchi, L., Thilker, D. A., Burgarella, D., et al. 2005, ApJ, 619, L71

Boselli, A., Lequeux, J., \& Gavazzi, G. 2002, A\&SS, 281, 127

Dolphin, A. E. 2000, PASP, 112, 1383

Dolphin, A. E., \& Kennicutt, R. C., Jr. 2002, AJ, 124, 158

Efremov, Yu. N. 1995, AJ, 110, 2757

Efremov, Yu. N. 2004, contribution to "Gamow-100" [arXiv:astro-ph/0410702] 
Elmegreen, B. G. 1994, ApJ, 433, 39

Elmegreen, B. G., \& Elmegreen, D. M. 1983, MNRAS, 203, 31

Elmegreen, B. G., \& Falgarone, E. 1996, ApJ, 471, 816

Elmegreen, B. G., \& Efremov, Y. N. 1997, ApJ, 480, 235

Elmegreen, B. G., Efremov, Yu. N., \& Larsen, S. S. 2000, ApJ, 535, 748

Elson, R. A. W., Fall, S. M., \& Freeman, K. C. 1987, ApJ, 323, 54

Fellhauer, M., \& Kroupa, P. 2002, AJ, 124, 2006

Fellhauer, M., \& Kroupa, P. 2005, MNRAS, 359, 223

Helfer, T. T., Thornley, M. D., Regan, M. W., et al. 2003, ApJSS, 145, 259

Henry, A. L., Quillen, A. C., \& Gutermuth, R. 2003, AJ, 126, 2831

Kennicutt, R. C. 1998a, ARA\&A, 36, 189

Kennicutt, R. C. 1998b, ApJ, 498, 541

Kennicutt, R. C., Lee, J. C., Funes, J. G., Sakei, S., \& Akiyama, S. 2004, in From 30 Doradus to Lyman break galaxies, ed. R. de Grijs, \& R. M. González Delgado Starbursts, in press

Kroupa, P. 1998, MNRAS, 300, 200

Larsen, S. S. 1999, A\&AS, 139, 393

Larsen, S. S. 2004, A\&A, 416, 537

Larsen, S. S., Efremov, Y. N., Elmegreen, B. G., et al. 2002, ApJ, 567, 896

Leitherer, C., et al. 1999, ApJS, 123, 3
Lejeune, T., \& Schaerer, D. 2001, A\&A, 366, 538

Maíz-Appelániz, J. 2001, ApJ, 563, 151

Mutchler, M., et al. 2005, Hubble Space Telescope multi-color ACS mosaic of M 51, the Whirlpool Galaxy, Bulletin of the American Astronomical Society, 37, 2

http://www. aas.org/publications/baas/v37n2/ aas206/339.htm

Rand, R. J. 1993, ApJ, 410, 68

Schmidt, M. 1959, ApJ 129, 243

Schweizer, F. 1976, ApJS, 31, 313

Solomon, P. M., Rivolo, A. R., Barrett, J., \& Yahil, A. 1987, ApJ, 319, 730

Scoville, N. Z., Polletta, M., Ewald, S., et al. 2001, AJ, 122, 3017

Strong, A. W., \& Mattox, J. R. 1996, A\&A, 308, L21

Whitmore, B. C., Zhang, Q., Leitherer, C., et al. 1999, AJ, 118, 1551

Williams, J. P., Blitz, L., \& Stark, A. A. 1995, ApJ, 421, 252

Wilson, C. D., Scoville, N., Madden, S. C., \& Charmandaris, V. 2003, ApJ, 599, 1049

Zhang, Q., Fall, S. M., \& Whitmore, B. C. 2001, ApJ, 561, 727

Zepf, S. E., Ashman, K. M., English, J., Freeman, K. C., \& Sharples, R. M. 1999, AJ, 118, 752

Zimmer, P., Rand, R. J., \& McGraw, J. T. 2004, ApJ, 607, 285 